\title{
A Conceptual Framework for Educational Design at Modular Level to Promote Transfer of Learning
}

\section{Botma Y}

School of Nursing, University of the Free State, Bloemfontein, South Africa

\author{
Van Rensburg, G. H.
}

Department of Health Studies, UNISA, Pretoria, South Africa

\author{
Coetzee, I. M.
}

Department of Nursing, University of Pretoria, Pretoria, South Africa

\section{Heyns, T.}

Department of Nursing, University of Pretoria, Pretoria, South Africa

Yvonne Botma, School of Nursing (99), P.O. Box 339, University of the Free State, Bloemfontein, South Africa, 9300. botmay@ufs.ac.za

$\mathrm{Y}$ Botma $(\mathrm{PhD})$ is an associate professor and lead researcher in innovative teaching and learning strategies in order to promote transfer of learning.

Gisela van Rensburg (D. Litt. et Phil) is a professor in nursing and lead researcher in support of students.

Isabel Coetzee (D. Litt. et Phil) does practice development in critical care nursing.

Tanya Heyns (D. Litt. et Phil) is the lead researcher in practice development. 


\begin{abstract}
Students bridge the theory-practice gap when they apply in practice what they have learned in class. A conceptual framework was developed that can serve as foundation to design for learning transfer at modular level. The framework is based on an adopted and adapted systemic model of transfer of learning, existing learning theories, constructive alignment, and the elements of effective learning opportunities. A convergent consensus-seeking process, which is typical of a qualitative approach, was used for expert review. The final conceptual framework consists of two principles: establishing a community of learning and the primacy of a learning outcome. The four steps entail: 1) activation of existing knowledge; 2) engaging with new information; 3) demonstrating competence; and 4) application in the real world. It is envisaged that by applying the framework educators in health care will design for transfer of learning, resulting in quality of care and optimal patient outcomes.
\end{abstract}

Keywords: conceptual framework; modular design; theory-practice gap; transfer of learning

\title{
Introduction
}

The unifying aim of all educators in health care is to enable students to render quality health care and to apply what they have learned in the classroom and simulation laboratories to real-world situations (Lauder, Sharkey, \& Booth, 2004). This process of application in the real world is known as "transfer of learning" or "theory-practice integration”. Holton, Bates, Bookter and Yamkovenko (2007) state that transfer of learning is the degree to which students apply to their jobs the knowledge, skills, behaviors, and attitudes they have gained in training. Transfer of learning is demonstrated by a competent student. Goudreau et al. (2009) summarise competency as follows “... thus allows one to deal with different situations by drawing on concepts, knowledge, information, procedures, and methods. It incorporates many elements, mobilizes knowledge, and strategically marshals capabilities in accordance with the specific nature of the situation" (p.3). Facilitation of problem solving, reflection, decision making, critical reasoning and relevant other skills is necessary to give students the opportunities to become competent (Jerlock, Falk, \& Severinsson, 2003). However, 
transfer of learning does not always occur. Many organisations have found that about $10 \%$ to $20 \%$ of training is ever applied in the real world (Ford, 2009; Kirwan \& Birchall, 2006). Failure to transfer learning occurs for several reasons and these may be grouped as factors within the student, design of the learning sessions, and organisational climate or workplace environment. The relationship of these factors is illustrated in Figure 1. Student characteristics, educational design, and workplace culture influence students' motivation to learn and to transfer learning and, in this way, performance in the clinical environment.

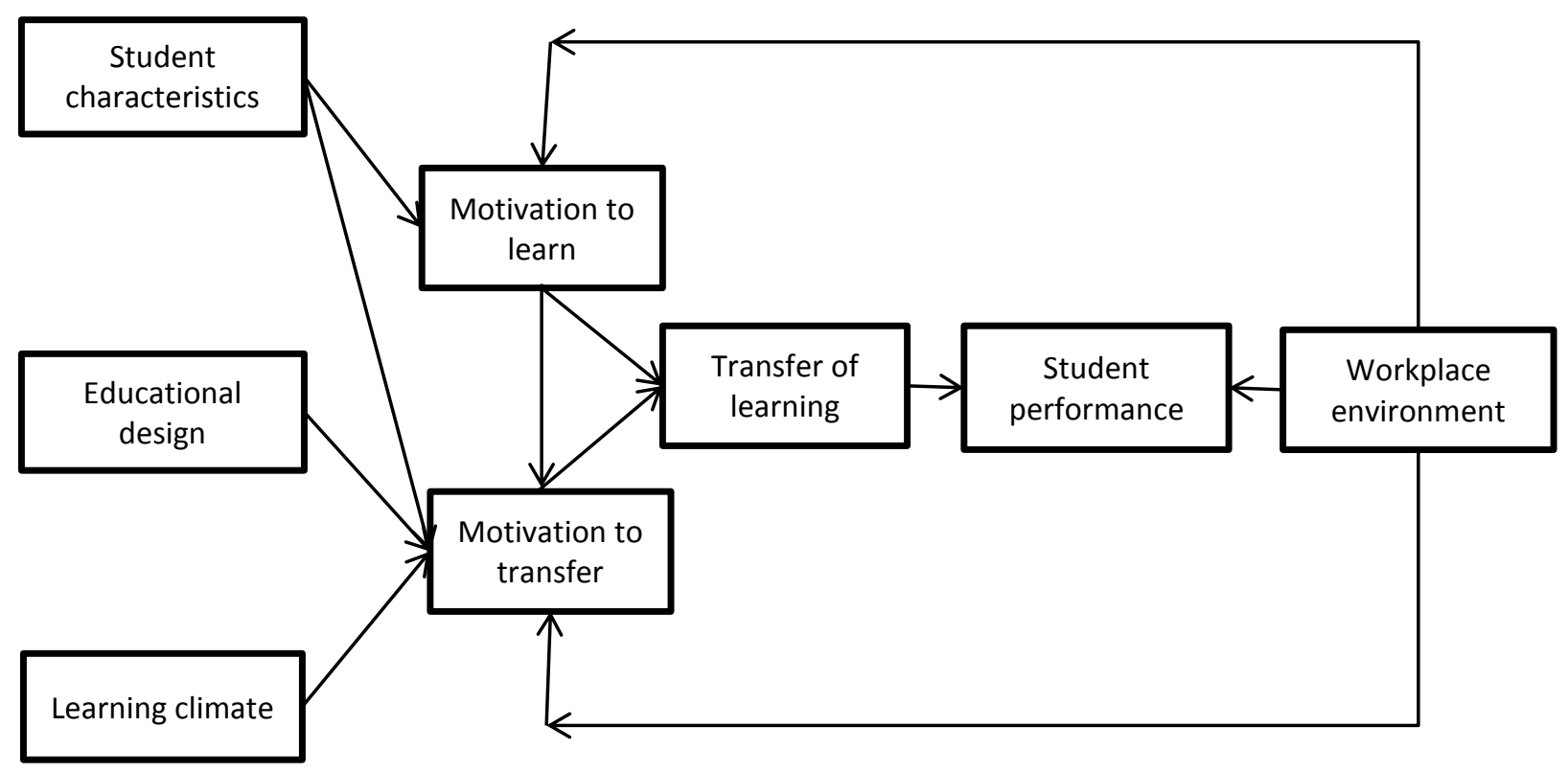

Figure 1: Systemic model of transfer of learning, adopted and adapted from Donovan and Darcy (2011)

In order to promote transfer of learning the educator must consider these factors when designing at modular level. For many educators it becomes a daunting task and they do not know where to start. It is the purpose of this article, therefore, to provide the conceptual framework that could serve as the foundation to design module themes in such a manner that it will promote transfer of learning.

\section{Methodology}

Developing conceptual frameworks involves identifying specific concepts, defining these concepts and then linking, integrating and aligning these to form a framework (Brink, Van Der Walt, \& Van Rensburg, 2006; Rycroft-Malone \& Bucknall, 2010). 
Initially, the first author of this article developed a conceptual framework of how to seamlessly incorporate simulation as a teaching and learning technique because the higher education institution she is affiliated with acquired authentic learning spaces with a number of human simulators.

Conceptual frameworks can be abstract, broad or skeletal in order to provide tapered information on a specific theme (Rycroft-Malone \& Bucknall 2010:27). An indepth literature review was conducted with regard to learning theories, competence, educational design and simulation. The literature review was later extended to include transfer of learning and/or learning and the theory-practice gap. In other words, the focus of the initial conceptual framework was changed from simulation to transfer of learning, because simulation is but one technique to promote transfer of learning. This framework provides a frame of reference to organise the thinking, problem solving and application needed in the clinical and non-clinical educational modules or themes.

The efficiency and usefulness of the conceptual framework is to assist and guide educators to describe the principles of a particular field to enhance the transfer of learning in a context. The use of the conceptual framework is set out in a systematic manner, which leads to the potential to explore and investigate the transfer of learning in different contexts (Smyth, 2004).

\section{Influential Theories}

Learning theories have reshaped themselves from behaviourism to information processing to constructivism. Recognising that context and culture influence memory and cognition forced researchers to explain personal meaning and the nature of reality and its representations (Doolittle \& Camp, 1999). Consequently social and radical constructivism developed with the following four principles:

- Learning is an active process according to which learners construct their own knowledge;

- Cognition is an adaptive process;

- Meaning making is a subjective process and does not render an accurate representation of reality; and

- Social, cultural, language, and biological/neurological processes influence knowing (Yilmaz, 2008). 
Educators usually have an eclectic approach to learning theories because they seldom support only one theory but consider instead what each has to offer. Therefore Piaget's view of learning, Vygotsky's theory of interactional learning, and Ausubel's concept of meaningful learning may all be considered relevant by an educator. Cognitive science and cognitive psychology confirm that the process of constructing knowledge is dependent on existing knowledge, the context or situation, and internalisation of information in an organised cognitive structure (Bruce, Klopper, \& Mellish, 2011). This constructivistic approach interrelates well with Kolb's experiential learning theory, which postulates that knowledge is created from understanding and transforming experience (Kolb \& Boyatzis, 2000). Kolb's model postulates that observations and reflections occur upon concrete experiences. These reflections are internalised and abstract concepts are formed with associated possible consequences of action. The possible associated consequences can be actively tested for validity and inform future actions in similar situations (Kolb \& Boyatzis, 2000). On the basis of these viewpoints of learning it is clear that the focus of learning outcomes has shifted from content to competence (Braband, 2008; Brandon \& All, 2010).

With the constructivist approach the educator becomes a facilitator of learning. For this reason the responsibility of the educator-facilitator is to create learning opportunities for students to process new information and link it to existing mental frameworks through individual or social activity. However, prior knowledge needs to be retrieved before the student is able to link the new knowledge to it. Through this process the new information is comprehended and meaning making occurs or knowledge is constructed.

Learning opportunities are created within a complex system that consists of the educator, student, teaching context, teaching and learning activities, outcomes, and student assessment tasks (Biggs, 1996) and should take the clinical environment into consideration. The starting point for creating learning opportunities is to clarify the learning outcome or competence (Biggs, 1996) that the professional person must demonstrate. Kouwenhoven defines competence as the capacity to demonstrate up to a predetermined standard the key occupational tasks that characterise a profession (Kouwenhoven, 2010.). According to Braband (2008), students are competent when they have the capacity to apply their knowledge and skills with an appropriate attitude in various environments and circumstances. In other words, when foundational 
knowledge (content), procedural knowledge (how to do), and conditional knowledge (when to do) become functional knowledge (Biggs, 1996).

The elements of learning opportunities that promote competence are activation of existing knowledge to serve as a foundation for new knowledge; application of knowledge in real-world settings; active engagement of students in real time and realworld situations; practicing of assessment, critical thinking, communication, and leadership skills through collaborative learning processes; multiple authentic formative assessments to ensure mastery of the complete competence; and objective assessment measures that are clearly aligned with expected competencies (Carraccio, Wolfsthal, Englander, Ferentz, \& Martin, 2002; Merrill, 2002). It is the responsibility of the educator to ensure that all teaching and learning activities are aligned with the outcome, reality, and assessment tasks while it is students' responsibility to actively engage with the learning material in order to internalise the theory and skills and in this way construct new knowledge (Reaburn \& Strategy, 2009).

\section{Development, Evaluation and Refinement of Conceptual Framework}

From an extensive literature review a conceptual framework was drafted by the first author, who is a member of a community of practice with a focus on scholarship of teaching and learning. This first author of this article presented the initial conceptual framework and supporting literature exploration to the rest of the team for a purposive review. In an attempt to refine and finalise the conceptual framework a process of expert review was utilised. The community of practice consists of educators in health care that have experience in: personal research, supervising postgraduate students, developing guidelines, and being active practitioners who facilitate learning.

The purpose of the critical review was to assess whether the framework could be: accepted as it was described; found acceptable but with recommendations for change or improvement; or found not to be acceptable at all. The group entered into a process of expert evaluation by critiquing the conceptual framework based on criteria set by Tastle, Wierman, and Dundum (2005). These criteria entailed:

- Clarity, simplicity and consistency - are constructs concrete and precise with clear descriptions?

- Appropriateness, relevance and comprehensiveness - are all aspects of transfer of learning addressed? 
- Applicability, practicality and usability - are there potential barriers in terms of implementation- and cost implications?

- Adaptability and transferability - are constructs transferable to various contexts and circumstances?

- Credibility - is the framework based on an extensive and critical literature review?

- Importance for research, practice and education - is the scientific value and contribution of the framework evident?

- Trustworthiness/validity - has a correct interpretation of the available evidence been drawn up to support the implementation of the framework?

To facilitate a well-informed review, a process was followed that involved reading, rereading and internalising the initial framework and supportive literature, followed by academic debate. The criteria listed immediately above were kept in mind. Agreement and consensus building are complex processes that require a thorough understanding of what is to be reviewed or assessed. The review was approached in a qualitative manner through a convergent process. Convergent processes include consensus formation regarding topics or aspects of topics that need to be addressed, their significance and the most effective means to address them. Tastle, Wierman and Dundum (2005) describe consensus as a function of shared team feelings towards an issue. Although these authors suggest a rating scale to capture these feelings [views] and to measure the extent to which a person agrees or disagrees with the issues put forward, the principle of consensus formation could also be applied to a qualitative approach to the review process.

The review discussions were based on an understanding of convergence among the members as experts and focused on the key constructs of a well-structured conceptual framework. These constructs were regarded as clear, appropriate, applicable, adaptable, practical, and credible and have value as proposed by Polit and Beck (2008). Polit and Beck's (2008) criteria are in accordance with those of Tastle, Wierman and Dundum (2005) that were initially used for review.The review process enabled the members of the community of practice to refine and finalise the conceptual framework. Changes were made to the extent of the framework so as not to limit it to the clinical environment. Focus was placed more on principles of transfer of learning rather than 
specific activities. It was agreed that these changes would make the framework more useful in a broader context of teaching and learning.

\section{Conceptual Framework}

On the basis of the principles of constructivism as a learning theory, constructive alignment and the elements of effective learning opportunities a conceptual framework was developed to guide educators on how to design themes for modules that would promote transfer of learning.

The conceptual framework consists of four steps - the activation of existing knowledge, engagement with new information, demonstration of competence, and application in real-world practice. (Refer to inner circle in Figure 2.) Criteria for successful implementation can be identified for each of these steps. (Refer to the four squares in Figure 2.) These four steps or phases are dependent on two principles - the primacy of learning outcomes and the demand that learning takes place within a community of learning. The four steps have as their objective the transfer of learning.

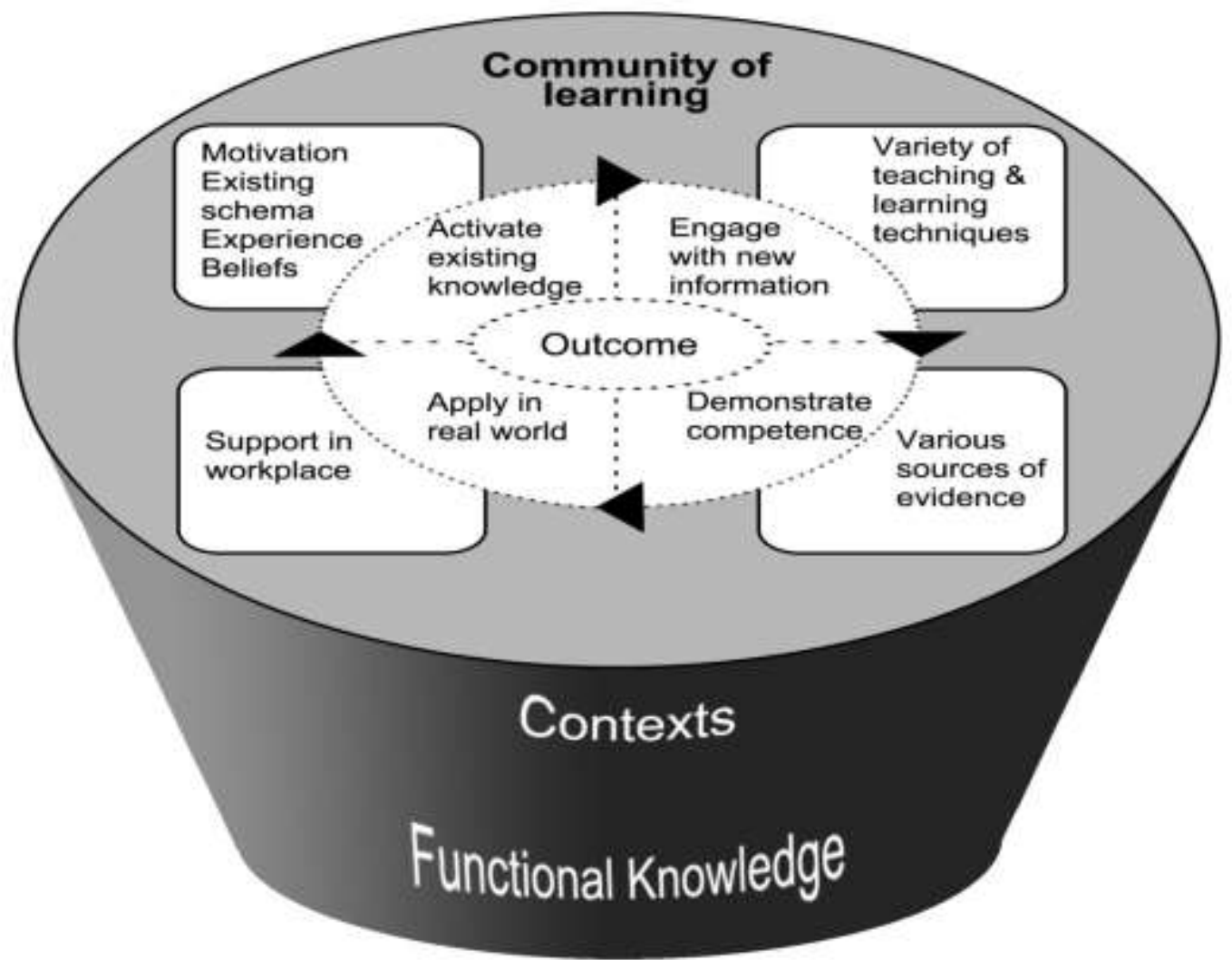

Figure 2. Conceptual framework for educational design at modular level to promote transfer of learning 


\section{Principles}

\section{Establish a community of learning}

Fellow students, facilitator(s), and experts in the field constitute a community of learning that assists in developing students' communication, critical thinking skills and the ability to elaborate and defend their views to others of different opinions (McLoughlin, 2001). Interaction amongst members of the learning community may occur face to face or through the use of electronic media (Reaburn \& Strategy, 2009; Kala, Isaramalai, \& Pohthong, 2010) and helps to build cognitive bridges (Brandon \& All, 2010). The quality and quantity of engagement and interaction are among other factors dependent on the degree of the presence of the facilitator (Raeburn, Muldoon, $\&$ Bookallil, 2009).

\section{The primacy of learning outcomes}

Researchers in cognitive psychology have shown that deep learning occurs when learners solve authentic problems, in such a way realizing the relevance of what they have learned (Merrill, 2002). For this reason, the educator should identify what a person at entry level into a profession should be able to do. Once that has been identified, it becomes the completion of the following stem sentence: "On completion of this module/theme the student will be able to ..."

\section{Four Steps}

\section{Step 1: activate existing knowledge}

As stated earlier, knowledge construction occurs when new information is integrated into existing mental schemas. Clark and Harrelson (2002) state that instructional events that activate relevant prior knowledge in long-term memory and stimulate internalisation in working memory supports knowledge construction. However, in practice many educators immediately start with new learning material without identifying the students existing knowledge. It is therefore essential to determine if the students have had any relevant experience and what that experience entailed. Information-orientated pre-tests are unproductive in activating prior experience (Merrill, 2002). In order to incorporate new information, existing schemas need to be recalled and modified. Existing knowledge and its related schema is often activated through 
story telling of lived experiences and guided reflection (Biggs, 1996). Doolittle and Camp (1999) state that it may be necessary to deconstruct an existing "incorrect" concept and then to (re)construct the appropriate concept before continuing with the desired teaching and learning activities (Doolittle \& Camp, 1999). This description fits with Mezirow's transformative learning as cited by Clapper (2010:e10); namely, that "it is a process of using a prior interpretation to construe a new or revised interpretation of one's experience in order to guide future action" (Clapper, 2010). The motivation to learn increases when students realise the relevance of the theoretical content.

\section{Step 2: engage with new information}

Learning is enhanced when the learning activities are aligned with the outcome or expected competence (Biggs, 1996). The engagement phase is student centered and outcome focussed because deep learning is promoted when students are actively engaged with the aim of making sense of the information by seeking integration between content and tasks (Pascoe \& Singh, 2008; Rust, 2002; Sefton, 2006). Students demonstrate engagement when they discuss information, think about it, or use and apply the information to solve real-life problems or challenges. Sefton (2006) recommends that students develop these skills progressively throughout the educational programme. Furthermore, students should know their learning style and preferred learning techniques to become life-long learners (Biggs, 1996; Pascoe \& Singh 2008; Boud \& Falchikov, 2006; Fink, 2003).

Content and skills should be pared down to what is essential to meet the learning outcome (Brandon \& All, 2010; Pascoe \& Singh., 2008). Forbes and Hicky (2009) advise that educators should ask themselves if the information will be used in the majority of situations in the specified context. When planning engaging learning activities, the facilitator should take into consideration that learning occurs best when more than one sense is involved - for example, when students see and hear (Clark \& Harrelson, 2002). Engagement should occur in all domains of learning and students should be offered opportunities to observe and practice how to perform certain skills correctly, because personal experiences are necessary for enhanced functioning within one's professional environment (Doolittle \& Camp, 1999). The emphasis during the engagement phase is on mastering content, becoming proficient in certain tasks and being actively engaged in the process of learning through various student-centered 
teaching methods. After the engagement phase students should be given the opportunity to demonstrate what they have learned.

\section{Step three: demonstrate competence}

Benner (2009) states that all learners within a professional programme progress from a novice- to competent practitioner. Novices are characterised by strict adherence to rules or plans with little situational perception and little ability to make discretionary judgments. At this level students need much guidance and support. At a competent level students have a broader understanding of the situation and can make more intuitive decisions (Roberts, Gustavs, \& Mack, 2012). Expertise is acquired through extensive involvement in activities that are specifically designed to improve performance (Ward, Hodges, Starkes, \& Williams, 2007). Educators should, however, recognise that this involvement is still part of the learning cycle and students also learn through their mistakes (Allan, Smith, \& O’Driscoll, 2011). When students are not skilled in what they need to do the specific incompetency should be identified and strategies implemented that give the student the opportunity to deliberately practice to become competent in those identifies areas (Clapper \& Kardong-Edgren, 2012).

\section{Step four: apply in real world}

Health care systems are workforce- and service orientated rather than learning oriented (Allan et al., 2011). Therefore, the workplace climate or organisational culture influences learning and is not always conducive to learning. Maben, Latter, and Clark (2006) describe the pressures and constraints of the system and professionals as organisational and professional sabotage. The sabotage may be due to time pressure, role constraints, staff shortage and poor skills mix, work overload, task orientation and high patient turnover. It is therefore essential to plan during the educational design phase how to support students in the workplace in order to enhance transfer of learning, thus applying it in the real world.

\section{Discussion}

Following the principles and the steps in this conceptual framework may enable students to apply what they have learned independently in different contexts over an extended period of time. In other words, the students will demonstrate their functional 
knowledge when they know when and how to use the content and skills that they have learned appropriately.

Known learning theories have been used to underpin the framework that could assist educators to design at modular level in order to enhance transfer of learning or bridge the well-described theory-practice gap (Allan, 2011; Maben et al., 2006). Although the initial conceptual framework was widely used in the institution where the first author works the refined conceptual framework needs to be applied outside that institution in clinical and non-clinical contexts. The rigour of the review process was enhanced by the fact that none of the experts are associated with the higher education institution of the first author. Furthermore, an independent skilled moderator facilitated the review process and thus reduced bias.

It is envisaged that by applying the framework, educators in health care will design for transfer of learning, resulting in quality of care and optimal patient outcomes. Furthermore, the principles and steps of the framework may culminate in a standardised educational design template used during the design process. This conceptual framework should become an integral part of curriculum development and could be applied in any professional educational context.

It is recommended that the conceptual framework be evaluated once it has been implemented to determine if transfer of learning occurred. Strategies to strengthen educators and clinical facilitators in applying the principles underlying the framework will become key to the value and contribution of this framework. Referring to the publication of Goetz and LeCompte (1984), Smyth (2004:168) states the conceptual framework can be utilised to guide and contribute to the methodological approach for future research in the transfer of knowledge.

\section{Conclusion}

In order to address the challenge that educators face in designing for transfer of learning the conceptual framework was developed that could serve as the foundation to educational design. This framework provides principles and steps that could assist in developing modular themes in such a manner that students are able to apply in practice what they have learned in theory. Existing mental schemas are recalled in preparation for the creation of new knowledge. Competence and expertise are developed within a community of learning during the engagement phase during which students are actively involved with learning activities that are well designed and aligned with the outcome 
and their learning needs. Students are afforded the opportunity to demonstrate their competence through various techniques of which simulation is an example. The framework further emphasises that learning in the workplace is part of the educational design and educators must consider how to support students in the workplace. Student support in the workplace can no longer be relegated to the service provider. The approach described in this article could assists educators in health care in applying overlapping principles of learning theories.

The principality of sound outcomes and a community of learning is emphasised in this conceptual framework. A health care professional needs to demonstrate functional knowledge which is the attainment of a sound learning outcome. As a result it is envisaged that successful application of this framework could cultivate a culture of lifelong learning that is a desired outcome for students studying for a professional degree.

\section{Financial disclosure}

The authors declare no conflict of interest.

\section{References}

Allan, H. T. (2011). Using psychodynamic small group work in nurse education: closing the theory-practice gap? Nurse education today, 31(5), 521-4. doi:10.1016/j.nedt.2010.09.006

Allan, H. T., Smith, P., \& O’Driscoll, M. (2011). Experiences of supernumerary status and the hidden curriculum in nursing: a new twist in the theory-practice gap? Journal of clinical nursing, 20(5-6), 847-55. doi:10.1111/j.13652702.2010.03570.x

Biggs, J. (1996). Enhancing Teaching through Constructive Alignment. Higher Education, 32(3), 347-364.

Boud, D., \& Falchikov, N. (2006). Aligning assessment with long-term learning. Assessment \& Evaluation in Higher Education, 31(4), 399-413. doi:10.1080/02602930600679050

Braband, C. (2008). Constructive alignment for teaching model-based design for concurrency. Lecture Notes on Computer Science, (2008), 1-18. 
Brandon, A., \& All, A. (2010). Constructivism theory analysis and application to curricula. Nursing Education Perspectives, 32(2), 89-92.

Brink, H., Van Der Walt, C., \& Van Rensburg, G. (2006). Fundamentals of research methodology for health care professionals (2nd ed.). Cape Town: Juta.

Bruce, J., Klopper, H., \& Mellish, J. (2011). Teaching and learning the practice of nursing (5th ed.). Cape Town: Pearson Education.

Carraccio, C., Wolfsthal, S. D., Englander, R., Ferentz, K., \& Martin, C. (2002). Shifting paradigms: from Flexner to competencies. Academic medicine : Journal of the Association of American Medical Colleges, 77(5), 361-7.

Clapper, T. C. (2010). Beyond Knowles: What Those Conducting Simulation Need to Know About Adult Learning Theory. Clinical Simulation in Nursing, 6(1), e7-e14. doi:10.1016/j.ecns.2009.07.003

Clapper, T. C., \& Kardong-Edgren, S. (2012). Using deliberate practice and simulation to improve nursing skills. Clinical Simulation in Nursing, 8(3), e109-e113. doi:10.1016/j.ecns.2010.12.001

Clark, R., \& Harrelson, G. L. (2002). Designing instruction that supports cognitive learning processes. Journal of athletic training, 37(4 Suppl), S152-S159.

Donovan, P., \& Darcy, D. P. (2011). Learning transfer: the views of practitioners in Ireland. International Journal of Training and Development, 15(2), 121-139. doi:10.1111/j.1468-2419.2011.00374.x

Doolittle, P., \& Camp, W. (1999). Constructivism: The career and technical education perspective. Vocational and Technical Education, 6(1), 1-16.

Fink, L. . (2003). A self-directed guide to designing courses for significant learning. San Fransisco: Jossey-Bass.

Ford, L. (2009). Improving training transfer. Industrial and Commercial Transfer, 41(2), 92-96. doi:10.1108/00197850910939135

Goudreau, J., Pepin, J., Dubois, S., Boyer, L., Larue, C., \& Legault, A. (2009). A second generation of the competency-based approach to nursing education. International journal of nursing education scholarship, 6(1), Article15. doi:10.2202/1548-923X.1685

Holton, E. F., Bates, R. A., Bookter, A. I., \& Yamkovenko, V. B. (2007). Convergent and divergent validity of the Learning Transfer System Inventory. Human Resource Development Quarterly, 18(3), 385-419. doi:10.1002/hrdq 
Jerlock, M., Falk, K., \& Severinsson, E. (2003). Academic nursing education guidelines: tool for bridging the gap between theory, research and practice. Nursing \& health sciences, 5(3), 219-28.

Kala, S., Isaramalai, S.-A., \& Pohthong, A. (2010). Electronic learning and constructivism: a model for nursing education. Nurse education today, 30(1), 61-6. doi:10.1016/j.nedt.2009.06.002

Kirwan, C., \& Birchall, D. (2006). Transfer of learning from management development programmes: testing the Holton model. International Journal of Training and Development, 10(4), 252-268. doi:10.1111/j.1468-2419.2006.00259.x

Kolb, D. A., \& Boyatzis, R. E. (2000). Perspectives on cognitive, learning, and thinking styles. In R. Sternberg \& L. Zhang (Eds.), NJ: Lawrence Erlbaum.

Kouwenhoven, W. (2010). Competence-based curriculum development in higher education : some African experiences. In M. Cantrell, M. Kool, \& W. Kouwenhoven (Eds.), Access \& Expansion: Challenges or Higher Education Improvement in Developing Countries (pp. 125-146). Amsterdam: VU University Press.

Lauder, W., Sharkey, S., \& Booth, S. (2004). A case study of transfer of learning in a family health nursing course for students in remote and rural areas. Nurse education in practice, 4(1), 39-44. doi:10.1016/S1471-5953(03)00017-9

Maben, J., Latter, S., \& Clark, J. M. (2006). The theory-practice gap: impact of professional-bureaucratic work conflict on newly-qualified nurses. Journal of advanced nursing, 55(4), 465-77. doi:10.1111/j.1365-2648.2006.03939.x

McLoughlin, C. (2001). Inclusivity and alignment: Principles of pedagogy, task and assessment design for effective cross-cultural online learning. Distance Education, 22(1), 7-29.

Merrill, M. D. (2002b). First principles of instruction. Educational Technology Research and Development, 50(3), 43-59. doi:10.1007/BF02505024

Pascoe, M., \& Singh, S. (2008). "By the end of this course you should be able to...": towards constructive alignment in the slp curriculum. The South African Journal of Communication Disorders, 55: 91-110.

Raeburn, P., Muldoon, N., \& Bookallil, C. (2009). Blended spaces, work based learning and constructive alignment: Impacts on student engagement. Proceedings Ascilite Auckland, 820-831. 
Roberts, T. V, Gustavs, J., \& Mack, H. G. (2012). Becoming an expert: a review of adult learning theory and implications for vocational training in ophthalmology. Clinical \& experimental ophthalmology, 40(5), 519-26. doi:10.1111/j.14429071.2011.02716.x

Rust, C. (2002). The Impact of Assessment on Student Learning: How Can the Research Literature Practically Help to Inform the Development of Departmental Assessment Strategies and Learner-Centred Assessment Practices? Active Learning in Higher Education, 3(2), 145-158. doi:10.1177/1469787402003002004

Rycroft-Malone, J., \& Bucknall, T. (2010). Models and frameworks for implementing evidence-based practice: linking evidence to action. Oxford: Blackwell Publishers.

Sefton, A. J. (2006). New approaches to medical education: an international perspective. Medical principles and practice : international journal of the Kuwait University, Health Science Centre, 13(5), 239-48. doi:10.1159/000079521

Tastle, W., Wierman, M. ., \& Dundum, U. . (2005). Ranking ordinal scales using the consensus measure. Issues in Information Systems, VI(2), 96-102.

Ward, P., Hodges, N. J., Starkes, J. L., \& Williams, M. a. (2007). The road to excellence: deliberate practice and the development of expertise. High Ability Studies, 18(2), 119-153. doi:10.1080/13598130701709715

Yilmaz, K. (2008). Constructivist Suggestions Constructivism: Its Theoretical Underpinnings, Variations, and Implications for Classroom Instruction. Educational Horisons, Spring, 161-172. 